\title{
A Study of the Difference in Operating Performance due to a Gaming Joystick's Styling and Button Locations
}

\author{
Chi-Hung Lo \\ Department of Industrial Design \\ Tunghai University \\ Taichung, Taiwan \\ chlo@thu.edu.tw
}

\author{
Ya-Chuan Ko \\ Department of Creative Product Design \\ Asia University \\ Department of Medical Research \\ China Medical University \\ Taichung, Taiwan \\ chrisko@asia.edu.tw
}

\begin{abstract}
In recent years, various types of operating interfaces for gaming have been developed accordingly due to the popularity of on-line games and gaming consoles. The psychological and physical burdens due to different types of operating interfaces are also different. Therefore, this study aims at the investigation of the difference in the operating difference of different styling designs and interfaces of gaming joysticks. The subject of ergonomics is to investigate the relationship between a user and the objects that he/she uses during his/her work or the environment from a human-centered point of view. It is required to pay attention to factors such as human capabilities, characteristics, and limitations for the design and application of an object. The ultimate goal is to guarantee the safety, efficiency, and comfort of people's life and work. From the ergonomic standpoint, this study is to investigate the operating performance and fatigue and burden indicators of different joystick interfaces, button functions, and joystick styling via real gaming tests and investigation by interviews. It was found in this study that, the most adequate button locations are on the left and right sides and these include top-bottom-left and top-bottom-right configurations. The optimal joystick styling should meet the recommended location for holding a joystick from the experimental results so that the fatigue due to using a gaming joystick can be reduced. The results of this study indicated that the second joystick among the three joystick designs in Experiment \#1 is the most ergonomics-compliant. Therefore, the second joystick was used for the investigation in Experiment \#2 for the matching of the location of graphical user interface (GUI) and the evaluation and correction of performance. The results indicated that the degree of eye fatigue is higher. By excluding the visual stimulus from the screen, it was not easy for users to understand or memorize the interface matching locations. As a result, users might easily generate the feeling of fatigue and the operating performance is not good.
\end{abstract}

Keywords—gaming joystick; ergonomics

\section{INTRODUCTION}

In advanced countries, the disorders caused by the musculoskeletal system has become the top one among all types of disorders. More than $40 \%$ of the compensations for occupational diseases in the US are related to the diseases related to the musculoskeletal system. Most of the preventive strategies for occupational musculoskeletal disorders are developed and performed by ergonomists. However, the results of literature review indicated that the real effects are very limited.

Based on the statistics by the Institute of Labor, Occupational Safety and Health, Ministry of Labor, Taiwan, musculoskeletal disorders account for about $60 \%$ of the injury or sickness benefits in the social security compensations and these include the low back pain and arm, neck, and shoulder disorders. The causes of these disorder include over-exertion, bad postures, overtime work, and high repetition. All of these make the body subject to big loading or cause fatigues due to repetitive fatigues. Starting from 2004, the cases of musculoskeletal disorders account for more than $90 \%$ of injury benefits of the social security in Taiwan. Among all types of disorders, the carpal tunnel syndrome (CTS) is the highest. For the proportions of different types of disorders related to the occupational musculoskeletal disorders in offices, disorders related to using computers account for $33 \%$ and disorders related to lifting up heavy objects account for $32 \%$ in addition to order typical office tasks as shown in Fig. 1.

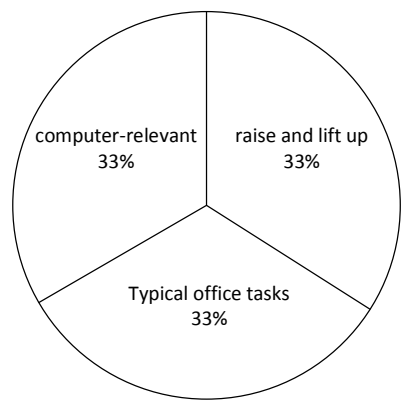

Fig. 1. Proportions of different types of occupational musculoskeletal disorders in offices

In the ergonomics field, the injuries related to the operating interface are composed of three factors which 
include exposure level, exposure frequency (i.e., repetition), and exposure time. Earlier ergonomic studies focused on the overload from the fitness point of view. However, a low level of long-term exposure and tasks that lack fitness activities such as offices tasks, computer operation tasks, and on-line games might cause hazards to health.

At the moment, most users use a joystick instead of a mouse for the operations with on-line games and video games. However, due to the difference in the styling and operation model of joysticks, a user might generate physical injuries due to operating a joystick for a long run. Typical disorders include the CTS, tendinitis or the mental burden due to the unhandy operations. It is expected to determine the operating model that is most suitable for typical consumers in this study. By determining the most suitable stick style for a joystick, the results serve as a good reference for a designer when designing a new joystick. The ultimate goal of this study is to assist a designer in creating joystick designs that reduce the physical and psychological burdens for a consumer during joystick operations.

\section{LITERATURE REVIEW}

The study of human-computer interaction (HCI) or computer-human interaction (CHI) is to understand people's way of using a computer system so as to design better systems that meet user demands. This type of studies aim to design a computer system that is safe, efficient, simple, and enjoyable with consummate functions [1].

There are four constituent elements for a $\mathrm{HCI}$ and they are described as follows.

1) User

2) A specific task or job that is required to be done

3) Under a specific context

4) Using a computer system

The fundamental conditions of a hand movement include coordination and stability. Studies in recent years indicated that a power grip is the main movement that causes workrelated musculoskeletal disorders (WMSDs) [2,3]. The types of fundamental functions of hands can be classified into five categories according to the difference in the figure locations and movement goals. These types include force touch, hook grip, precision handling, grasp, and power grip. The common movements that occur during the usage of a joystick include finger force touch and palm handling.

For the design principles of hand tools, Fraser [4] proposed a stick styling design that follows the movement pattern of hand grip. Moreover, Selan [5] also recommend an arc pattern for the design of the stick for a hand tool. For example, the surface profile of the recess should follow the shape of four fingers and the surface profile of the protrusion should meet the shape of the palm and the thumb root. Studies in recent years indicated that a stick design should emphasize more on the relationship between a stick and a palm. For example, a curved stick presents more operating performance on the push force and it has a higher level of comfort [6].

A bad hand tool design causes cumulative trauma disorders (CTDs) which can be classified into three fundamental patterns including tendon injuries (i.e., tendonitis, tenosynovitis, DeQuervain's disease), nerve injuries (i.e., carpal tunnel syndrome, Guyon tunnel syndrome), and nerve vessel injuries (i.e., thoracic outlet syndrome, vibrationinduced white finger). The possible causes to these injuries include bad wrist and shoulder posture, excessive hand force, repeated movements with a high frequency [7].

For the measurement of the subjective ranking of hand tools, the local perceived discomfort (LPD) evaluation is often used. It emphasizes on the degree of discomfort for the arm and hand. The portions from the hand to the wrist are divided into 12 regions and the arm is divided into 2 regions. This approach evaluates the perception of pains, the degree of paralysis and stress, and the fatigue caused by discomfort. A six-point scale is used for evaluating the degree of discomfort (0: not discomfort; 5: complete discomfort) [8].

The sales of a Japanese brand, AFC, in Taiwan indicated that the best seller among all types of dietary supplements for the last two years are the marigold and lutein. These two supplements are advocated as a good solution that helps reduce high eye pressure and dry eye issues. This phenomenon indicates the trend of more and more readings and internet surfing for modern people. It also reflects the need of more product designs that could reduce a user's eye fatigue such as the improved design of a computer screen or manual operation interface. A computer's quality can be improved from the physical aspect so as to create designs that are comfortable and easy for a user to understand.

The investigations in this study consist of in-depth observations by experiment and questionnaire surveys which include three types of investigation as follows

\section{B. Human body measurement}

The palm of each of the research subjects was measured and their preferences for the joystick size were investigated in order to conclude the curvature and size of each palm and adequate size of a joystick for a user to hold. The corresponding comfort indicators can be obtained [9].

\section{Grip force experiment}

This experiment is to measure the pressure distribution of each of the joysticks on different portions of a palm. The results of the grip force experiment on the ergonomic handles that were created were compared with the subjects' subjective ranking so as to determine their operating performance and comfort degrees [10].

\section{Interface experiment}

This experiment is to determine the optimal joystick button layout and interface which is easy to operate and could reduce a user's learning stress [11].

\section{RESEARCH PROCEDURE}

The procedure of this study is described as follows. 


\section{A. Investigation from literature review}

Earlier studies that are relevant to the design guidelines for hand tools or hand muscle portions were collected and reviewed. Criteria that are related to the measurement of hand tools were also reviewed.

\section{B. Experiments of this study}

The experiments of this study were carried out by the following three steps.

- The first step is to categorize the fundamental dimensions of the subjects' palms by real measurements on human bodies.

- The second step is to investigate the muscular loading of the forearm, upper arm and the back muscle when holding different joysticks. The subjects were asked to rank the joysticks during the subjective questionnaire survey. The regions on a palm with uncomfortable feelings can be determined. The range of palm pressure during operation can be reasonably and objectively inferred.

- The third step is to select a suitable joystick from Experiment \#1 for the investigation of operating interface in Experiment \#2. The goal is to determine the joystick button layout and interface that reduce a user's learning stress and is easy to operate.

\section{Conclusion and recommendation}

The results of this study were summarized so as to propose our recommendations for joystick designs. These results serve as a good reference for a designer in this industry to create optimal joysticks.

\section{RESEARCH SCOPE AND LIMITATIONS}

The research scope of this study and its limitations are described as follows.

- Since there is a wide range of joysticks for use as hand tools, only the joysticks that are used for gaming were investigated in this study.

- There models of the most commonly seen joysticks on the market were selected as the specimens for investigation in this study.

- The interviews were carried out on site. During the interviews with the subjects, the interview contents might be affected by the subjective views of the subjects and the operators. This phenomenon might limit the objectivity of the interview contents and this is another limitation of this study.

- The spatial range of this study is within the Tainan area in Taiwan.

\section{RESEARCH SUBJECTS}

The research subjects of this study are described as follows Based on the earlier studies of human body measurements on palm sizes, each of the male and female groups was divided into two subgroups by $25 \%$ and $50 \%$ of the total palm length. There are a total of 8 subjects including 4 females and 4 males. The typical height of males is in the range of $175 \sim 180 \mathrm{~mm}$ and that of females is in the range of $150 \sim 165 \mathrm{~mm}$. The subjects are within the age bracket of 16 26 years old with an average age of 23.625 years old.

\section{RESEARCH METHODOLOGY}

The experimental approach was used in this study and the popular on-line game PLAN-S was selected as the platform for carrying out the joystick tests. The experimental contexts consists of two parts. The first part is to investigate and compare the degree of fatigues that are caused by different joystick styling designs. The second part is to verify whether different button locations cause the difference in the achievement rate during operation of the experiment. In Experiment \#1, the joystick comfort and ergonomic dimensions were investigated. The main goal is to determine the difference in the areas and locations that are subject to forces for different joysticks. The procedure is described as follows.

1) Select three different gaming joysticks that are commonly used on the market

2) Use a pape tape to wrap the gaming joysticks and apply pigments to the paper tape. Ask a research subject to wear gloves for gaming tests

3) Carry out the tests with same songs

4) Gather and summarize the data of the area and range of pressure distribution on the glove for further analysis

TABLE I. SAMPLES OF GAMING JOYSTICKS THAT ARE COMMONLY SEEN ON THE MARKET

\begin{tabular}{|l|c|c|c|}
\hline Joystick \# & 1 & 2 & 3 \\
\hline Front view & 8 & \\
\hline Side view & &
\end{tabular}

In Experiment \#2, investigations on different button models were carried out to obtain the degree of completeness. The goal is to find out the button interface that is most suitable for the game. The procedure is described as follows.

1) Select the optimal gaming joystick from Experiment \#1

2) Select one song and ask each of the subjects to carry out operations on four different interfaces

3) Summarize the suitable button configurations based on the degree of completeness

A comparison of different button configurations is shown in Table 2. 
TABLE II. DIFFERENT BUTTON CONFIGURATIONS

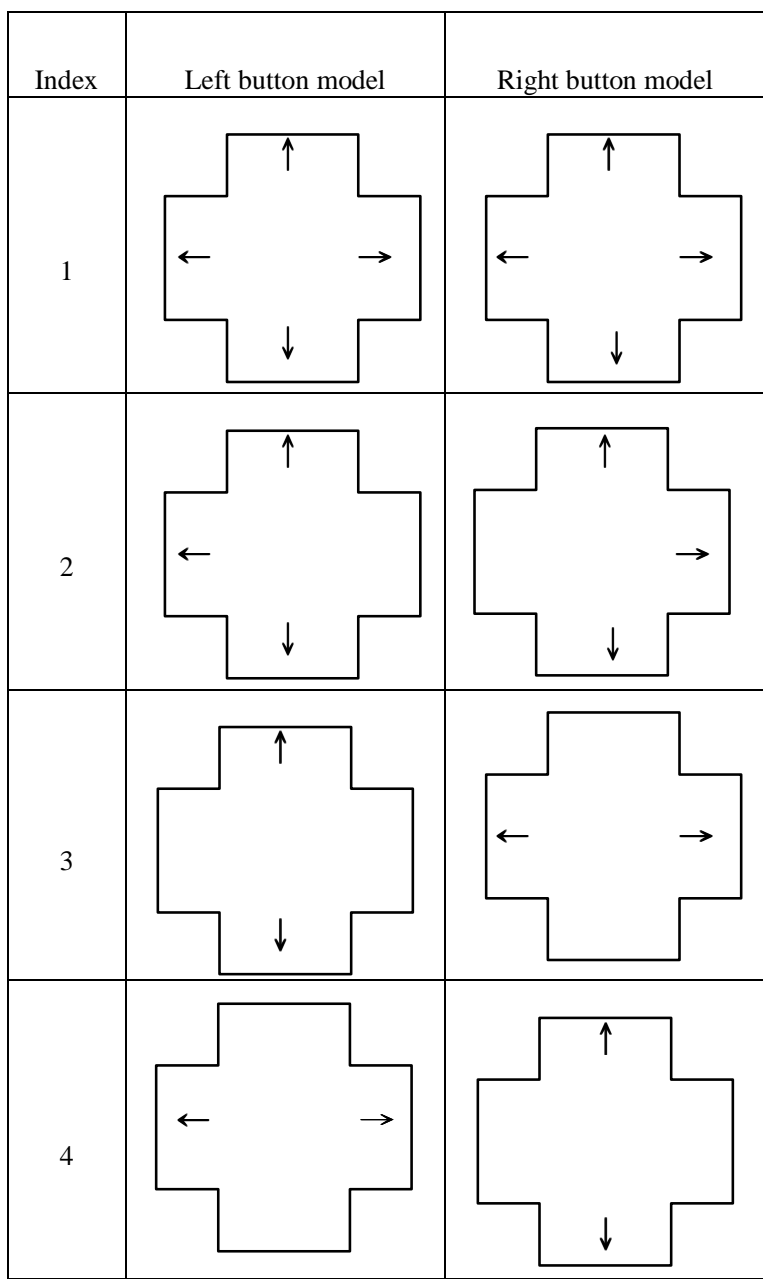

VII. RESULTS AND DISCUSSIONS

The results of the subjective ranking of the operation completeness in Experiment \#1 were obtained from a questionnaire survey. The second joystick design was determined to be the most adequate one among those three joysticks. The results of the LPD evaluation indicated that the top three areas with the feeling of soreness are respectively Index A: The first knuckle of the thumb; Index D: Palm center; Index I: The second knuckle of the index finger on the right hand. Similarly, the top three areas with the feeling of soreness on the left hand are respectively Index A: The first knuckle of the thumb; Index B: First dorsal interosseous; Index D: Palm center. For the degree of body loading, the top three areas with fatigue and soreness are respectively Index 8: Palm; Index 7: Wrist; Index 1: Eyes as shown in Fig. 2 and Fig. 3.

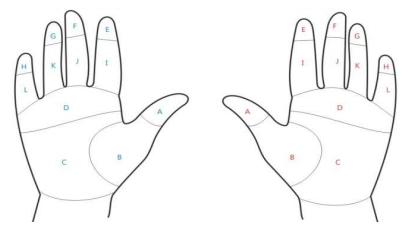

Fig. 2. Different portions of a palm for LPD evaluation

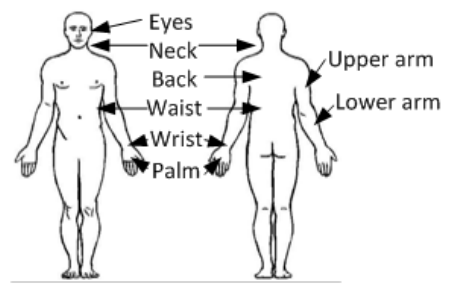

Use a number in the range of 1 7 to indicate the fatigue index of each region. 1 indicates the lowest degree of fatigue; 7 indicates the highest degree of fatigue.

Fig. 3. LPD evaluation

In Experiment \#2, Button Model \#2 has the highest degree of achievement rate for the overall operation and Button Model \#4 has the lowest achievement rate. The results of the subjective comparison by the subjects indicated that Button Model \#2 is the optimal button model as shown in Table 3.

TABLE III. ACHIEVEMENT RATE OF DIFFERENT BUTTON MODELS

\begin{tabular}{|c|c|c|c|c|}
\hline Index & 1 & 2 & 3 & 4 \\
\hline Achievement rate & $61.85 \%$ & $62.525 \%$ & $49.267 \%$ & $45.903 \%$ \\
\hline
\end{tabular}

For the objective measurements, the area and range of the glove are summarized to obtain the locations which were subjected to forces. The glove was scanned in the second portion of the experiment to obtain a flat glove mark. By using the Photoshop software, the transparency of the images was adjusted so that images can be stacked for comparison in order to find the locations of a palm being subjected to forces as shown in Table 4.

TABLE IV. COMPARISON OF THE PRESSURE DISTRIBUTION ON THREE JOYSTICK DESIGNS

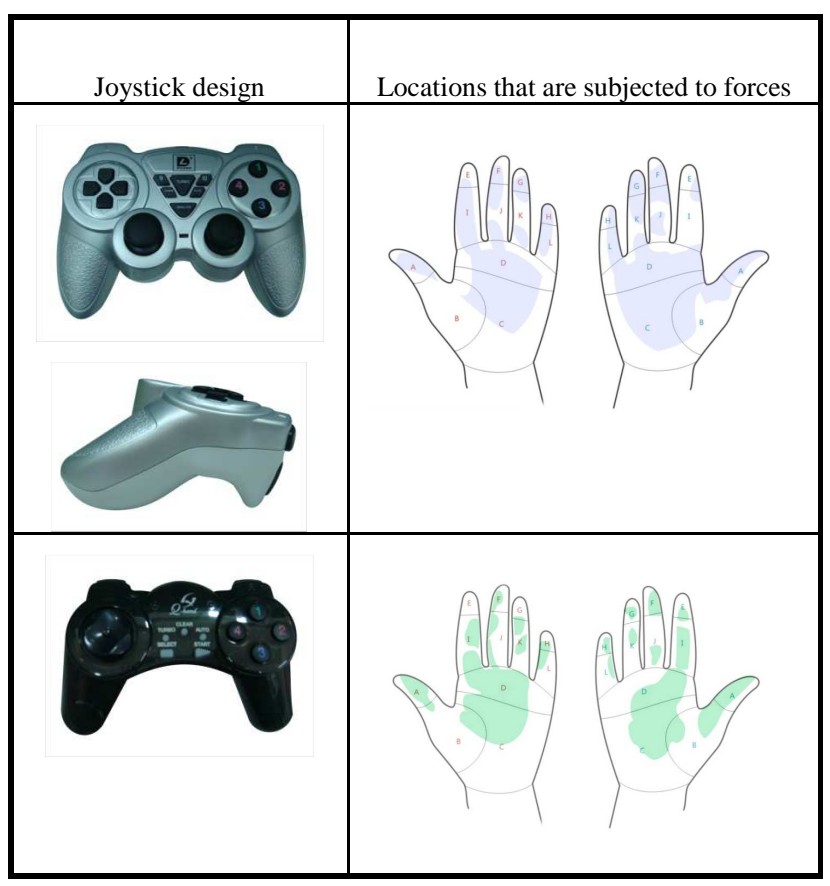




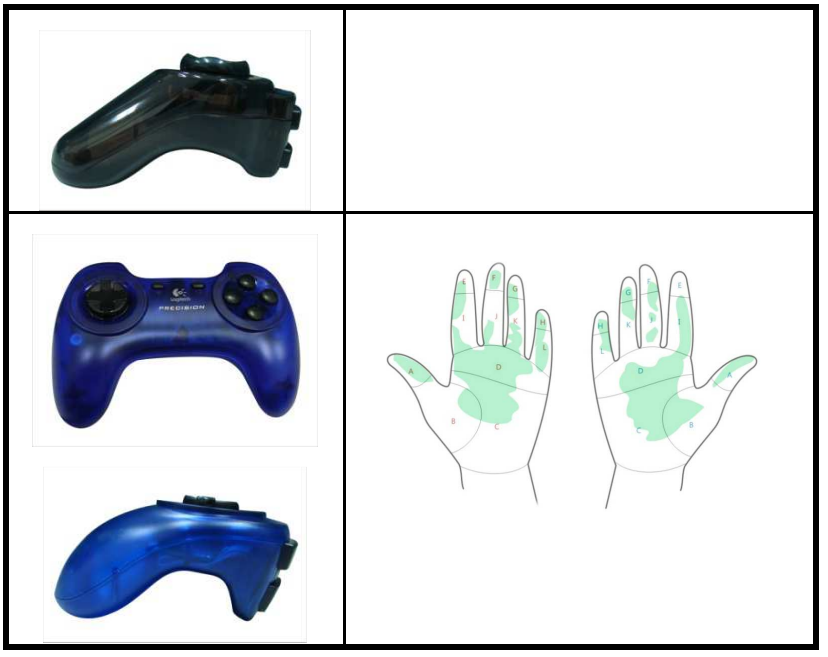

For the button interface designs in Experiment \#2, the model of Index \#2 is the easiest to operate and understand, followed by Index \#1. The most difficult one to operate is Index \#4 as shown in Fig. 4.
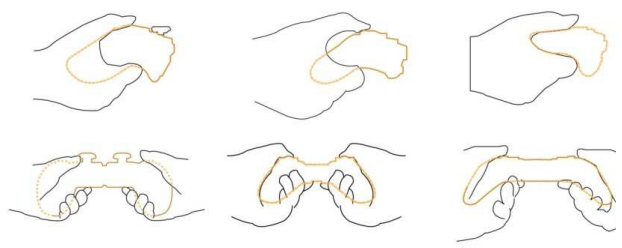

Fig. 4. Locations of pressure distribution on three joystick designs

\section{CONCLUSION AND RECOMMENDATIONS}

The results of the palm pressure distribution indicated the following findings. (1) The palm pressure of a female is about $80 \%$ of that of a male. (2) The pressure at the thumb portion of a palm is the highest, followed by the first dorsal interosseous. (3) From the styling point of view, the pressure of the Index \#2 joystick has the smallest pressure, followed by Index \#3. The pressure of Index \#1 is the highest. (4) From the standpoint of button interface design, the configuration of Index \#2 is the easiest to operate and understand, followed by Index \#1. Index \#4 is the most difficult to operate. The results of this investigation also proposed the grouping the button allocations and the simplification of relative correlations for easy identification.

The results also indicated that, most of the forces exert on the central area of a palm. Sanders \& McCormick (1993) [12] also indicated that this portion is where the important vessels, particularly the ulnar artery, radial artery, and nerves are located. This region is very sensitive to pressure. Therefore, for the design of this region on a joystick, it is advised to use soft materials instead of hard materials so as to enhance a user's comfort for long-term use. For the region on a joystick for handling, it should be designed with a wider area for contact so that the pressure can be distributed to a larger region. For the interface design, follow-up studies are advised to investigate further on the relevant games with different colors to highlight or with vibration hints for guidance when a user conducted a wrong operation.

\section{Acknowledgment}

The authors would like to thank the Ministry of Science and Technology of Taiwan, ROC for the financial support under grant number MOST 105-2221-E029-011.

\section{References}

[1] J. Preece, "A guide to usability-human factor in computing", 1998.

[2] E.Y. Chao, F. Opgrandi, and M. Axmere, "Three dimensional force analysis of the finger joints in selected isometric hand functions," J Biomech, 1976, pp. 387-396.

[3] B. Buchholz, L.J. Frederick, and T.J. Armstrong, " An investigation of human palmar skin friction and the effects of materials, pinch force and moisture," Ergonomics, 31(3), 1988, pp.317-325.

[4] T.M. Fraser, "Ergonomics design of hand tools, encyciopedia of occupational health and safety," International Labour Office, Geneva, vol.1, no.3, 1983.

[5] J.L. Selan, "The Advanced Ergonomics Manual," Dallas: Advanced Ergonomics Inc., 1994.

[6] P.H. Cheng, "The ergonomic design and evaluation of handsaw," 2006.

[7] W.Y. Yeh and Y.W. Lin, "A study of the feasibility of ergonomic checklist for on-site works," Bureau of Labor Insurance, Ministry of Labor, 1995.

[8] L. Groenesteijn, S.M. Eikhout, P. Vink, "One set of pliers for more tasks in installation work: the effects on (dis)comfort and productivity," Appl. Ergon, 35, 2004, pp.485-492.

[9] L.F.M. Kuijt-Evers, T. Bosch, M.A. Huysmans, M.P. de Looze, and P. Vink, "Association between objective and subjective measurements of comfort nd discomfort in hand tools," Applied ergonomics, vol.38, 2007, pp.643-654.

[10] M.L. Lu, T. James, B. Lowe, M. Barrero, and Y.K. Kong, "An investigation of hand forces and postures for using selected mechanical pipettes," International Journal of Industrial ergonomics, vol.38, 2008, pp.18-29.

[11] S.P. Wu and C.S. Hsieh, "Ergonomics study on the handle length and lift angle for the culinary spatula," Applied Ergonomics, vol.33, 2002, pp.493-501.

[12] M.S. Sanders and E.J. McCormick, Human Factors In Engineering and Design, 7th ed., New York: McGraW-Hill, 1993, pp.417-418. 